\title{
Widely Separated Deformations of Singular Potential
}

\author{
Sylwia Kondej \\ Institute of Physics, University of Zielona Góra, \\ ul. Szafrana 4a, 65-246 Zielona Góra, Poland \\ e-mail: skondej@proton.if.uz.zgora.pl
}

(Received: 23 March 2010; accepted 8 July 2010; published online: 6 September 2010)

\begin{abstract}
We study the two dimensional quantum system governedby the Schrödinger operator with delta type potential. The interaction is supported by line $\Gamma$ which coincides with a straight at infinity and which admits two widely separated deformations. The aim of this paper is to express the number of bound states of our system by the number of bound states of the system with single deformation.
\end{abstract}

Key words: singular potential, bound states

\section{INTRODUCTION}

\section{Formulation of the problem}

The paper belongs to the research often called singular perturbation; for rich bibliography see [1]. The potentials in this kind of models are localized on sets of lower dimensions. More specifically, we are interested in the two dimensional quantum system governed by the Schrödinger operator with attractive delta type potential supported by an infinite line $\Gamma$. Then the Hamiltonian $H_{\alpha \Gamma}$ of such system can be intuitively written as

$$
-\Delta-\alpha \delta(x-\Gamma), \alpha>0 ;
$$

the precise definition of $H_{\alpha \Gamma}$ will be discussed in the next section. It was shown in [2] that if $\Gamma$ is not straight but asymptotically straight, then $H_{\alpha \Gamma}$ has at least one eigenvalue below the threshold of the essential spectrum. Let us recall here that the essential spectrum of $H_{\alpha \Gamma}$ takes the form

$$
\sigma_{\mathrm{ess}}\left(H_{\alpha \Gamma}\right)=\left[-\frac{\alpha^{2}}{4}, \infty\right)
$$

In [3] the scattering problem was discussed for the system governed by $H_{\alpha \Gamma}$. This paper is addressed to the problem of the number of bound states of $H_{\alpha \Gamma}$. However, we consider here a special geometry of $\Gamma$ which we precisely describe below.

\section{Geometry of $\Gamma$}

Suppose $\Gamma$ is an infinite $C^{4}$ curve in $\mathbb{R}^{2}$ which does not admit self-intersections. Let $\Sigma$ stand for a straight line in $\mathbb{R}^{2}$. Assume that $\Gamma$ admits two disconnected widely separately deformations, i.e.

$$
\Gamma \backslash \Sigma=\Lambda_{1} \cup \tilde{\Lambda}_{1}
$$

where $\Lambda_{1}, \tilde{\Lambda}_{1}$ are finite and disconnected curves and put $d$ for the distance between them, i.e. $d=\inf |x-\tilde{x}|$ for $x \in \Lambda_{1}, \quad \tilde{x} \in \tilde{\Lambda}_{1}$. Putting in mind the latter purposes, it is useful to introduce $\Lambda_{0}$ and $\widetilde{\Lambda}_{0}$ so that

$$
\Sigma \backslash \Gamma=\Lambda_{0} \cup \tilde{\Lambda}_{0},
$$

and $\Lambda_{i} \cup \tilde{\Lambda}_{i}, i=0,1$ form loops. Then $\Gamma_{*}=\left(\Sigma \Lambda_{0}\right) \cup \Lambda_{1}$ forms a line with one deformation determined by $\Lambda_{1}$. Similarly, we define $\tilde{\Gamma}_{*}=\left(\Sigma \backslash \tilde{\Lambda}_{0}\right) \cup \tilde{\Lambda}_{1}$ with deformation supported by $\widetilde{\Lambda}_{1}$ only.

\section{The main result}

It is naturally to think that for $d \rightarrow \infty$ the system in the same sense decomposes to two separated systems governed by $H_{\alpha \Gamma_{*}}$ and $H_{\alpha \tilde{\Gamma}_{*}}$. For example, it appears the question whether the spectrum $\sigma\left(H_{\alpha \Gamma}\right)$ of $H_{\alpha \Gamma}$ approaches $\sigma\left(H_{\alpha \Gamma_{*}}\right) \cup \sigma\left(H_{\alpha \tilde{\Gamma}_{*}}\right)$ for $d \rightarrow \infty$. The partial answer to this question concerning the number of eigenvalues from the discrete spectrum states the main result of this paper, namely we have 


$$
\sharp \sigma_{d}\left(H_{\alpha \Gamma}\right)=\sharp \sigma_{d}\left(H_{\alpha \Gamma^{*}}\right)+\sharp \sigma_{d}\left(H_{\alpha \tilde{\Gamma}^{*}}\right) .
$$

Moreover, the eigenvalues of $H_{\alpha \Gamma}$ approach the eigenvalues of $H_{\alpha \Gamma_{*}}$ and $H_{\alpha \tilde{\Gamma} *}$ for $d \rightarrow \infty$.

Let us mention here that some upper bounds for the number of bound states of $H_{\alpha \Gamma}$ were already derived in [4]. It was shown, for example, that

$$
\sharp \sigma_{d}\left(H_{\alpha \Gamma}\right) \leq 1+F\left(\alpha, c, L, k_{m}\right),
$$

where $c$ is a constant satisfying $|\Gamma(s)-\Gamma(t)| \leq c|s-t|$ and $\Gamma(\cdot): \mathbb{R} \mapsto \mathbb{R}^{3}$ is the arc length parameterization of $\Gamma, L$ denotes the length of minimal component $\Pi$ of $\Gamma$ so that $\Gamma \backslash \Pi \subset \Sigma$ and $k_{m}$ is the maximal curvature of $\Gamma$. Function $F$ was discussed in detail in [4]. However, the above upper bound does not reflect the equivalence (1) for widely separated deformations. Therefore, for this model it is more optimal to combine both results, i.e.

$$
\sharp \sigma_{d}\left(H_{\alpha \Gamma}\right) \leq 2+F_{\Gamma_{*}}+F_{\tilde{\Gamma}_{*}},
$$

where $F_{\Gamma *}$ defines upper bound corresponding to $H_{\alpha \Gamma_{*}}$, cf. (2), and analogously $F_{\tilde{\Gamma}_{*}}$ corresponds to $H_{\alpha \tilde{\Gamma}_{*}}$.

\section{DEFINITION OF HAMILTONIAN AND ITS RESOLVENT}

We are interested in Hamiltonian of the two dimensional quantum system which can be intuitively written as

$$
-\Delta-\alpha \delta(x-\Gamma), \alpha>0,
$$

where $\Gamma$ is a line in $\mathbb{R}^{2}$ with geometry described in the previous section. The Hamiltonian corresponding to (3) can be defined by means of the following quadratic form

$$
\begin{gathered}
\varepsilon_{\alpha \Gamma}(\psi, \varphi)=\int_{\mathbb{R}^{2}} \overline{\nabla \psi}(x) \nabla \varphi(x) \mathrm{d} x-\alpha \int_{\mathbb{R}} I_{\Gamma} \bar{\psi}(s) I_{\Gamma} \varphi(s) \mathrm{d} s, \\
D\left(\mathcal{E}_{\alpha \Gamma}\right)=W^{2,1}\left(\mathbb{R}^{2}\right),
\end{gathered}
$$

symbol $I_{\Gamma}$ denotes here the embedding $W^{2,1}\left(\mathbb{R}^{2}\right) \mapsto L^{2}(\mathbb{R})$, i.e. $I_{\Gamma} \psi=\psi * \delta_{\Gamma}$, where $\delta_{\Gamma}$ is the Dirac function with the support on $\Gamma$. Using the results of [5] one can show that $\mathcal{E}_{\alpha \Gamma}$ defines a unique self-adjoint operator in $L^{2}\left(\mathbb{R}^{2}\right) \equiv L^{2}$. The resulting operator we denote $H_{\alpha \Gamma}$.

\section{Resolvent of $H_{\alpha \Gamma}$}

To construct the resolvent $R_{\Gamma}^{\kappa}:=R_{\alpha \Gamma}^{\kappa}=\left(H_{\alpha \Gamma}+\kappa^{2}\right)^{-1}$, $\kappa>\alpha / 2$ we are following the idea derived in [3]. We give here a summary of this construction omitting some technical details studied in [3]. Let $R_{\Sigma}^{\kappa}$ stand for resolvent of Hamiltonian with straight line interaction. Moreover, put $v_{i}$ and $\widetilde{v}_{i}, i=0,1$ for the Dirac measures on $\Lambda_{i}$ and $\widetilde{\Lambda}_{i}$, resp. Let us introduce

$$
\mathcal{H}=\mathrm{h} \oplus \tilde{\mathrm{h}},
$$

where $\mathrm{h}=L^{2}\left(v_{0}\right) \oplus L^{2}\left(v_{1}\right)$ and analogously for the tilde symbols. Standardly, we introduce the embeddings $R_{\Sigma, v}^{\kappa}: \mathrm{h} \mapsto L^{2}, \quad\left(R_{\Sigma, v}^{\kappa}\right)^{*}: L^{2} \mapsto \mathrm{h} \quad$ and $\quad R_{\Sigma, v v}^{\kappa}: \mathrm{h} \mapsto \mathrm{h}$. We construct the analogous embeddings for space $\widetilde{\mathrm{h}}$ obtaining $\left(R_{\Sigma, \widetilde{v}}^{K}\right), \quad\left(R_{\Sigma, \widetilde{v}}^{\kappa}\right)^{*}, \quad R_{\Sigma, \widetilde{v} \widetilde{v}}^{K}$ and 'mixed' embeddings $R_{\Sigma, v \widetilde{v}}^{\kappa}, R_{\Sigma, \widetilde{v} v}^{\kappa}$.

Now we are ready to define so called Birman-Schwinger operator acting in $\mathrm{h} \oplus \widetilde{\mathrm{h}}$ :

$$
\begin{gathered}
\Theta^{\kappa}=-\left(\begin{array}{cc}
-\alpha^{1} \breve{\mathrm{I}}_{v}+R_{\Sigma, v v}^{\kappa} & R_{\Sigma, \nu \tilde{v}}^{\kappa} \\
R_{\Sigma, \tilde{v} v}^{\kappa} & -\alpha^{1} \breve{\mathrm{I}}_{\tilde{v}}+R_{\Sigma, \tilde{v} \tilde{v}}^{\kappa}
\end{array}\right) \\
\breve{\mathrm{I}}_{v}=\left(\begin{array}{cc}
\mathrm{I} & 0 \\
0 & -\mathrm{I}
\end{array}\right)
\end{gathered}
$$

where

and $\breve{\mathrm{I}}_{v}$ acts on $L^{2}\left(v_{0}\right) \oplus L^{2}\left(v_{1}\right)$; analogously we define $\breve{\mathrm{I}}_{\widetilde{v}}$. For $-\kappa^{2} \in\left(-\infty,-\alpha^{2} / 4\right) \backslash \sigma_{d}\left(H_{\alpha \Gamma}\right)$ the operator $\Theta^{\kappa}$ is invertible with the bounded inverse. Consequently, the resolvent of $H_{\alpha \Gamma}$ takes the form, cf. [3],

$$
R_{\Gamma}^{\kappa}=R_{\Sigma}^{\kappa}+R_{\Sigma, \mu}^{\kappa}\left(\Theta^{\kappa}\right)^{-1}\left(R_{\Sigma, \mu}^{\kappa}\right)^{*}, \quad \text { where } \mu=v+\widetilde{v} .
$$

\section{Remark 1}

Similarly, one can show that the resolvents of $H_{\alpha \Gamma_{*}}$ and $H_{\alpha \tilde{\Gamma} *}$ take the forms

$$
\begin{gathered}
R_{\Gamma *}^{\kappa}=R_{\Sigma}^{\kappa}+R_{\Sigma, v}^{\kappa}\left(\Theta_{v}^{\kappa}\right)^{-1}\left(R_{\Sigma, v}^{\kappa}\right)^{*}, \\
R_{\tilde{\Gamma} *}^{\kappa}=R_{\Sigma}^{\kappa}+R_{\Sigma, \tilde{v}}^{\kappa}\left(\Theta_{\tilde{v}}^{\kappa}\right)^{-1}\left(R_{\Sigma, \tilde{v}}^{\kappa}\right)^{*}, \\
\Theta_{v}^{\kappa}=\alpha^{1} \breve{\mathrm{I}}_{v}-R_{\Sigma, \tilde{v} \tilde{v}}^{\kappa}, \Theta_{\tilde{v}}^{\kappa}=\alpha^{1} \breve{\mathrm{I}}_{\tilde{v}}-R_{\Sigma, \tilde{v} \tilde{v}}^{\kappa} .
\end{gathered}
$$

where

\section{THE MAIN RESULT}

To prove the main statement, we will relay on the Birman-Schwinger principle which says

$$
-\kappa^{2} \in \sigma_{d}\left(H_{\alpha \Gamma}\right) \quad \text { iff } \quad \operatorname{ker} \Theta^{\kappa} \neq\{\emptyset\} .
$$

Theorem 1. For d large enough we have

$$
\sharp \sigma_{d}\left(H_{\alpha \Gamma}\right)=\sharp \sigma_{d}\left(H_{\alpha \Gamma_{*}}\right)+\sharp \sigma_{d}\left(H_{\alpha \Gamma_{*}}\right) .
$$

Moreover, the eigenvalues of $H_{\alpha \Gamma}$ approach the eigenvalues of $H_{\alpha \Gamma_{*}}$ and $H_{\alpha \widetilde{\Gamma}_{*}}$ for $d \rightarrow \infty$. 
Proof. We provide here only a sketch of proof omitting some standard technical details.

Using the explicit forms for $R_{\Sigma, v \widetilde{v}}^{\kappa}$ and $R_{\Sigma, \tilde{v} v}^{\kappa}$, cf. [3], one shows by a straightforward calculation that both nondiagonal term of $\Theta^{K}$ goes to 0 in the norm operator sense if $d \rightarrow \infty$. Therefore we have

$$
\Theta^{\kappa}=\Upsilon^{\kappa}+E^{\kappa}(d), \quad \Upsilon^{\kappa}=-\left(\begin{array}{cc}
\Theta_{v}^{\kappa} & 0 \\
0 & \Theta_{\tilde{v}}^{\kappa}
\end{array}\right)
$$

and $E^{\kappa}(d)$ goes to 0 in the norm operator sense for $d \rightarrow \infty$. statement from Remark 1 we get

$$
\operatorname{ker} \Upsilon^{\kappa} \neq\{\emptyset\} \quad \text { iff } \quad-\kappa^{2} \in \sigma_{d}\left(H_{\alpha \Gamma_{*}}\right) \cup \sigma_{d}\left(H_{\alpha \tilde{\Gamma}_{*}}\right) \text {. }
$$

Suppose that $\Upsilon^{\kappa_{0}} \varphi_{0}=0$ and $\varphi_{0}$ is normalized. Since $\Theta^{\kappa}$ satisfies pseudo-resolvent equivalence for $\kappa$ close to $\kappa_{0}$ we get

$$
\Theta^{\kappa}=\left(-\kappa^{2}+\kappa_{0}^{2}\right)\left(\cdot, \varphi_{0}\right)_{\mathcal{H}} \varphi_{0}+A^{\kappa}+E^{\kappa}(d),
$$

where $A^{\kappa}=\mathcal{O}\left(\left(\kappa^{2}-\kappa_{0}^{2}\right)^{2}\right)$ in the norm operator sense. Using the above equivalence we state that $\operatorname{ker} \Theta^{\kappa} \neq\{\varnothing\}$ iff there exists a solution of

$$
\eta(\kappa, d):=\left(\left(A^{\kappa}+E^{\kappa}(d)\right) \varphi_{0}, \varphi_{0}\right)_{\mathcal{H}}+\kappa_{0}^{2}-\kappa^{2}=0 .
$$

Applying the implicit function theorem to $\eta$ can show that $\operatorname{ker} \Upsilon^{\kappa_{0}} \neq\{\varnothing\}$ iff there exists $\breve{\kappa}_{0}$ so that $\operatorname{ker} \Theta^{\breve{\kappa}_{0}} \neq\{\varnothing\}$ for $d \rightarrow \infty$ and $\breve{\kappa}_{0} \rightarrow \kappa_{0}$. This, in view of (4), states the claim.

\section{References}

[1] S. Albeverio, F. Gesztesy, R. Høegh-Krohn, H. Holden, Solvable Models in Quantum Mechanics. 2nd printing (with Appendix by P. Exner), AMS, Providence, R.I. (2004).

[2] P. Exner, T. Ichinose, Geometrically induced spectrum in curved leaky wires. J. Phys. A34, 1439-1450 (2001).

[3] P. Exner, S. Kondej, Scattering by local deformations of a straight leaky wire. J. Phys. A38, 4865-4874 (2005).

[4] J. Cisło, S. Kondej, Upper bound for the number of bound states induced by the curvature of singular potential. submitted.

[5] J.F. Brasche, P. Exner, Yu.A. Kuperin, P. Šeba, Schrödinger operators with singular interactions. J. Math. Anal. Appl. 184, 112-139 (1994).

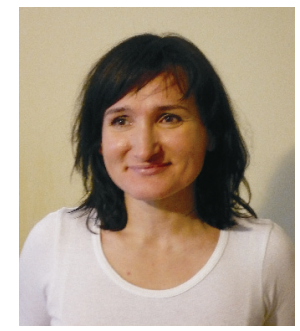

DR. SYLWIA KONDEJ graduated in mathematical physics from Wroclaw University in 1996 and received the $\mathrm{PhD}$ degree from the Wroclaw University in 2001. She was a postdoc at the Czech Academy of Science in Rez (near Prague) and got Emmy Noether position at the Technische Universitat in Chemnitz. Since 2003 she has been Assistant Professor at the Institute of Physics of Zielona Gora University. Her research interest is focused on so called singular perturbations of Schroedinger operators. 